\author{
Karsten Garscha \\ (Frankfurt am Main)
}

\title{
EUROPA UND LATEINAMERIKA BEI ALEJO CARPENTIER. EINE POLEMISCHE REVISION
}

I.

Es mag erstaunen, dass ich mich mit einer Thematik zu Wort melde, über die schon so viel und vielleicht auch Endgültiges gesagt wurde. Doch ich bin im Zusammenhang eines Seminars nach längerer Zeit einem meiner früheren Lieblingsautoren wieder begegnet und konnte mich dabei des Gefühls einer großen Ernüchterung, ja Enttäuschung, nicht erwehren: Es handelt sich um Alejo Carpentier und seinen Roman El Recurso del Método.

Dieser Beunruhigung nachzugehen, schien mir passend auf einem Forschungskolloquium, dessen Titel »Selbstvergewisserung am Anderen oder Der fremde Blick auf das Eigene « heißt und dessen Untertitel von »Diskursen interkultureller Erfahrung und deren Inszenierung in den Literaturen Spanischamerikas« spricht.

Alle in der Themenstellung des Kolloquiums enthaltenen Begriffe stehen im Zentrum des gesamten Erzählwerks des kubanischen Romanciers:

1. Lateinamerika wird in Carpentiers Texten so gut wie immer in Opposition zu Europa zur Anschauung gebracht; sie haben häufig eine geradezu dichotomische Struktur (>acá</ >allá<).

2. Niemand unter den lateinamerikanischen Autoren spricht so konsequent von interkultureller Erfahrung wie Carpentier.

3. Und niemand gibt seinen Texten so sichtbar die Form von Inszenierungen und von literarischen Konstruktionen.

Es scheint mir legitim und überdies notwendig, dem Blick Carpentiers auf Europa und auf Lateinamerika heute kritisch zu begegnen. Und in gewisser Weise gilt das nicht nur für ihn, sondern für die gesamte nueva novela, ja 
für die lateinamerikanische Literatur überhaupt. Nachdem sie unbestritten ein Teil der Weltliteratur ist, sind die Zeiten der Apologie ${ }^{1}$ vorbei; jetzt geht es darum, eine kritische neue Lektüre und Revision dieser Literatur vorzunehmen. Dafür gibt es eine Reihe von Gründen; die drei folgenden Faktoren scheinen mir besonders wichtig:

1. Der Begriff der Nationalliteratur, der schon immer eine zweifelhafte Konstruktion war, löst sich auf. Immer häufiger steht einer in den Grenzen eines Landes geschriebenen Literatur eine im Exil entstandene gegenüber; das 20. Jahrhundert bietet hierfür zahlreiche Beispiele (Deutschland, Spanien, Kuba, Chile, Kamerun, Kongo usw.). Unzählige Autoren leben im >Ausland Sprachen; denken wir etwa an Hector Bianciotti, der in die Académie Française aufgenommen wurde, an Guillermo Cabrera Infante, der sich des Englischen, oder an Jorge Semprún, der sich des Französischen bedient. Blicken wir auf Afrika, so verfasst kaum einer der bekannten Autoren seine Texte in seiner >Muttersprache<; fast alle leben in Paris, London, New York oder Montreal. Das heißt, alle Literaturen sind transnational und transkontinental vernetzt; sie bilden, im Zeitalter der mondialisation, textuell ebenso wie wirtschaftlich, eine > Weltliteratur ${ }^{2}{ }^{2}$

2. Eine substanzielle, wesenhafte Begründung von Andersartigkeit wie bei Carpentier trägt nicht mehr. An die Stelle des Ideals eines mestizaje cultural tritt die Vorstellung von culturas hibridas; die ,Wurzel< als Metapher für Identität wird von der des >Rhizoms` abgelöst.

3. Offensichtlich sind mit dem Ende des 20. Jahrhunderts auch die klassischen Ideologien und Utopien an ihr Ende gekommen.

1 Eine solche apologetische Grundtendenz findet sich zum Beispiel noch in der von Michael Rössner (1995) herausgegebenen Lateinamerikanische[n] Literaturgeschichte. Cf. dazu meine Besprechung in der Frankfurter Rundschau (1996). - Ich verweise an dieser Stelle auch auf meinen Beitrag zum Berliner Kolloquium Europäische Avantgarde im lateinamerikanischen Kontext (1989), wo ich "Alejo Carpentiers Verhältnis zur europäischen, besonders zur französischen Avantgarde« skizziert habe; dort argumentiere ich pro, hier contra Carpentiers Denkmuster; cf. Karsten Garscha (1991).

2 Siehe hierzu die hochinteressante Studie von Pascale Casanova (1999). 
II.

Werfen wir nun, gut fünfundzwanzig Jahre nach seinem Erscheinen, einen Blick auf El Recurso del Método, und lassen wir uns dabei von der Frage leiten, welches Bild von Europa und von Lateinamerika Carpentier in diesem Roman entwirft. Dessen Protagonist ist ein lateinamerikanischer Despot, der als Primer Magistrado in Paris als aufgeklärter und gebildeter Präsident gilt und in den besten Kreisen verkehrt, in Lateinamerika aber seine diktatorische Herrschaft nur mit brutaler Gewalt gegen Aufstände und Putsche aufrecht erhalten kann.

Europa, das ist in diesem Roman Frankreich oder eigentlich nur Paris, die von allen lateinamerikanischen Dichtern und Intellektuellen geliebte Kulturhauptstadt der Welt. Neben Frankreich und Paris steht Descartes, dessen Discours de la Méthode jedem Kapitel des Buches ein Motto liefert und durch einen Silbentausch dem Roman auch seinen Titel beschert. Diese Reduzierung Europas auf Paris und auf den französischen Zentralismus und Rationalismus ist typisch für Carpentiers gesamtes Werk, auch wenn andere europäische Länder - Spanien, Italien, Deutschland oder Rußland durchaus vorkommen. In El Recurso del Método befinden wir uns in der ,ville-lumière des Fin de Siècle, der Belle Époque und des Ersten Weltkriegs, im Paris von Marcel Proust, dessen Figuren - die Verdurins, Elstir, Raynaldo Hahn, Forcheville oder Brichot - uns hier leibhaftig begegnen. Dieses Paris repräsentiert Europas Dekadenz, und für Carpentier gehören Dekadenz und Europa untrennbar zusammen:

Y a Francia le vendría bien un sacudimiento, una terapéutica de emergencia, un shock, para sacarla de un autosuficiente letargo. Harto engreída, necesitaba una lección. Demasiado rectora del mundo se creía aún, cuando, en realidad, agotadas sus grandes energías, había entrado en una fase de evidente decadencia. Había terminado el Reino de los Gigantes: Hugo, Balzac, Renán, Michelet, Zola. $(\mathrm{RM}, 109)^{3}$

3 Siglen der zitierten Primärliteratur in der Reihenfolge ihrer Besprechung: $\mathrm{RM}=$ El Recurso del Método, in: Carpentier, Alejo (1984): Obras completas, Bd. VI, Mexiko: Siglo XXI; REM = El Reino de este Mundo, in: Carpentier, Alejo (1983): Obras completas, Bd. II, Mexiko: Siglo XXI; CP = La Consagración de la Primavera, in: Carpentier, Alejo (1978): Obras completas, Bd. VII, Mexiko: Siglo XXI; PP = Los Pasos perdidos, in: Carpentier, Alejo (1983): Obras completas, Bd. II, Mexiko: Siglo $\mathrm{XX1}$. 
Lateinamerika hingegen strotzt vor Vitalität: Das zeigt sich an seiner wilden und mächtigen Natur, an seinen Gebirgen, seinen Sümpfen, der maßlosen Hitze und den verheerenden Stürmen, das drückt sich aus im Monumentalismus seiner Bauten, das manifestiert sich in seiner barbarischen, ursprünglichen Grausamkeit, das erkennt man an der ausladenden Rhetorik seiner Sprache oder am feurigen Geschmack seiner Speisen. In Amerika verwandelt sich der Primer Magistrado in einen gewaltigen Haudegen und Macho, der das Bett mit der Hängematte vertauscht und dessen probates Mittel zur Lösung von Problemen die nackte Gewalt ist.

Diese Andeutungen genügen, um zu zeigen, dass wir es hier mit einer Schwarz-Weiß-Zeichnung zu tun haben, die vollständig aus Klischeevorstellungen besteht und wie eine Karikatur wirkt. Das exotistische Lateinamerikabild könnte einem Hollywood-Film entnommen sein, in dem ein barbarischer Tyrann sein Land unterjocht. Die Akkumulation von Namen, Titeln und Zitaten bei der Beschreibung von Paris ist nichts weiter als ein oberflächlicher Enzyklopädismus, der an keiner Stelle in die Tiefe dringt und jedes Gespür für die Radikalität und Experimentierfreude etwa der klassischen Moderne vermissen lässt.

Carpentiers Ziel ist einzig und allein die systematische Abwertung Europas und die Aufwertung Lateinamerikas, mit der expliziten didaktischen Intention, die Notwendigkeit des heroischen Kampfes der kubanischen Kommunisten gegen die Diktatoren Machado und Batista zu begründen, der seine historische Erfüllung im »Triunfo de la Revolución« von 1959 gefunden hat. Aus heutiger Sicht (und wenige Tage nach dem Tod von Herberto Padilla) erhält der antieuropäische Tenor des Buches noch eine besonders pikante Note; denn 1974 hatten die meisten europäischen Intellektuellen und Schriftsteller ihrer Begeisterung für den Máximo Lider ein plötzliches Ende gesetzt, nachdem der »Caso Padillo« gezeigt hatte, wie es um die Meinungsfreiheit auf Kuba in Wahrheit bestellt war. Gewiss, El Recurso del Método ist eines der schwachen Bücher Carpentiers, und es hält den Vergleich nicht aus mit Roa Bastos' Yo el Supremo über Doctor Francias Entwicklung vom Aufklärer zum Diktator oder mit dem kürzlich erschienenen Politkrimi La Fiesta del Chivo, in dem Vargas Llosa Trujillos grausame Herrschaft und seine Ermordung evoziert. Darum wollen wir das Verhältnis von Europa und Lateinamerika in seinen anderen Romanen kurz beleuchten. 
III.

El Reino de este Mundo, Carpentiers erster bedeutender literarischer Versuch aus dem Jahre 1949, kennzeichnet bereits der schroffe Gegensatz zwischen Europa und Lateinamerika. Diesen Antagonismus entwickelt Carpentier in seinem programmatischen Vorwort über die Ästhetik des real maravilloso. Diese besteht im Wesentlichen aus einer maßlos überzogenen Polemik gegen den Surrealismus, der als künstliche, phantasielose und dekadente Kunstrichtung verhöhnt wird, weit entfernt vom wahren, pulsierenden Leben. Die Surrealisten seien nichts als trickreiche Taschenspieler, und dies gelte vor allem für Breton. Dagegen setzt er den Vitalismus Lateinamerikas, der sich im lebendigen Glauben, in der exuberanten Natur, im Reichtum der sich mischenden Kulturen und in den im Volk lebendigen Mythen manifestiere. Dabei entnimmt Carpentier alle zentralen Begriffe seiner Ästhetik den surrealistischen Manifesten, behauptet aber, nur Lateinamerika besitze die Voraussetzungen, die es einem schöpferischen Geist erlaubten, die von den Surrealisten intendierte Kunst auch wirklich zu schaffen.

Es liegt auf der Hand, wie grotesk Carpentier den Surrealismus und die historischen Avantgarden missversteht und verzerrt, im Gegensatz zur avantgardistischen Musik, mit der er sich höchst fruchtbar und intensiv auseinandersetzt. Breton ist für ihn wie die Reinkarnation von Descartes, und er sieht im Surrealismus so etwas wie die Wiedergeburt und den Inbegriff des arroganten französischen Cartesianismus mit seinen unberechtigten universalistischen Ansprüchen.

Besonders an zwei Stellen zeigt sich, zu welch sonderbaren Formulierungen ihn seine Faszination von der Vitalität Lateinamerikas hinreißt. In der Aufzählung der Wesensmerkmale Lateinamerikas findet sich auch die Wendung »por la presencia fáustica del indio y del negro" (REM, 17). Hier geht Carpentier in seinem Überschwang einiges durcheinander; denn wer könnte seuropäischer sein als Faust? Wenige Abschnitte davor scheut er sich nicht, ein Abscheu erregendes Zitat von Lautréamont noch zu übertrumpfen: 
Hay todavía demasiados adolescentes que hallan placer en violar los cadáveres de hermosas mujeres recién muertas> (Lautréamont), sin advertir que lo maravilloso estaría en violarlas vivas. (REM, 14)

El Reino de este Mundo präfiguriert in gewisser Weise Barnets Cimarrón, und stellt, vor dem Hintergrund des Afrokubanismus, des haitianischen Indigenismus und der Négritude, die Sklavenaufstände, den Kampf um die Unabhängigkeit Haitis und die verdrehte Welt Henri Christophes aus der Sicht des schwarzen Sklaven Ti Noel dar. Die weißen Plantagenbesitzer sind schwächliche, versoffene und dekadente Figuren, deren Rassismus die Aufstände auslöst. Um den unüberbrückbaren Antagonismus zwischen der afrikanischen, auf mündlicher Überlieferung basierenden und der europäischen, der Schrift verpflichteten Kultur möglichst eindringlich vorzuführen, greift Carpentier im ersten Abschnitt des zweiten Kapitels von El Reino de este Mundo zu Racines Phèdre. Es ist natürlich kein schärferer Gegensatz denkbar als zwischen der hochstilisierten Sprache Racines und den raffinierten Regeln der höfischen Ästhetik der französischen Klassik auf der einen und der magisch-mythischen Wahrnehmungweise der schwarzen Sklaven auf der anderen Seite. So inszeniert und so unwahrscheinlich diese Szene auch ist (denn wie sollten diese Menschen Racines sublime Verse verstehen?), sie erlaubt es Carpentier, wie mit Paukenschlägen die Artifizialität des europäischen Theaters der Unmittelbarkeit des Voodoo, den rein literarischen antiken Mythos dem alles erfassenden Glauben der Afroamerikaner, die Deklamation der Authentizität, kurz: die der Lebenswirklichkeit ferne europäische Kunst der Intensität und Umfassenheit des Lebens der afrikanischen Sklaven gegenüberzustellen.

Wie Descartes repräsentiert hier die französische Klassik ein Europa, das vor lauter Fiktionen längst den Kontakt zum eigentlichen Leben verloren hat und das seine Ästhetik, seine Sprache, seine Denkformen der ganzen Welt aufzwingen möchte. Die Sklaven kennen den europäischen Begriff der Kunst gar nicht. Alles Künstlerische hat einen bestimmten Platz im Ablauf ihres Lebens, und so vermögen sie es nicht, Racines Verse als etwas lediglich Imaginiertes wahrzunehmen. So gewaltig diese Passage auch wirken mag, so alt und so abgedroschen ist die Polemik gegen das

4 Dieser Satz erregt immer wieder den besonderen Zorn meiner Studentinnen und Mitarbeiterinnen, die diesen frauenverachtenden Aspekt des maravilloso vehement zurückweisen und das Frauenbild, das Carpentier zeichnet, scharf kritisieren. 
weltfremde, lebensferne, artifizielle und langweilige Theater Racines. Die deutschen Dramatiker des 18. Jahrhunderts haben sich dieser Argumente reichlich bedient, und Victor Hugo tut es ihnen gleich, wenn er in der Préface de Cromwell Lope de Vega und Shakespeare gegen Racine und die Einheitsregeln ausspielt. Es kommt Carpentier überhaupt nicht darauf an, Europa und Lateinamerika auf gleichem Niveau zu konfrontieren. Das Urteil über Europa ist längst gefällt. Vergessen wir nicht, dass es zu Lebzeiten Ti Noels in Europa bereits einen Montesquieu, einen Voltaire und einen Diderot gab, und dass diese Autoren auch, wenngleich mit Schwierigkeiten, nach Lateinamerika gelangten. Eine komplexere Diskussion und Verarbeitung der Problematik wechselseitigen Verstehens und Missverstehens entspricht freilich nicht Carpentiers didaktischer und saufklärerischer< Intention.

Eberhard Geisler hat in einem ebenso abgewogenen wie scharfsinnigen Aufsatz schon 1985 nachgewiesen, auf wie reduzierte und klischeehafte Weise Carpentier gegen Ende seines Lebens in La Consagración de la Primavera erneut gegen Breton und den Surrealismus zu Felde zieht. ${ }^{5}$ Die sein ganzes Werk strukturierende Europa-Lateinamerika-Opposition wird hier aufs Neue variiert:

Debo decir que toda esa faramalla de conceptos, objeciones, teorías, abjuraciones, controversias, guerras de cafés (La Coupole contra el Cyrano, Les DeuxMagots contra Le Palmier [...]) se me estaba volviendo de una increíble frivolidad frente a los dramas reales y cruentos que se vivían en América Latina. Aquí se asistía, en estos momentos, a una pugna, de labios para afuera, entre quienes aceptaban las consignas de un partido revolucionario, los que aspiraban a un posible maridaje de revolución y poesía, y los que, queriendo mostrarse revolucionarios a toda costa se iban por los ya socorridos disparaderos del trotsquismo y del anarquismo donde nada se exigía a nadie, salvo una insaciable capacidad de decir «no», unida a la tenacidad del perro del hortelano. Aquí, se hablaba de una sangre posible; allá, la sangre enrojecía las aceras. Aquí, se hablaba de actuar; allá, se actuaba, y, harto a menudo, por actuar se moría. Aquí, se firmaban ma-

5 Cf. Eberhard Geisler (1985: 6): "Breton aparece como charlatán que en lugar de seguir un trabajo honrado pierde el tiempo en los cafés, jactándose, además, de un elitismo barato. Con esto, Carpentier le niega casi toda la importancia al trabajo intelectual y pasa por alto también el hecho de que el surrealismo haya puesto en duda el valor del trabajo como fundamento de la sociedad occidental, valor que el marxismo había dejado intacto. El contexto de esta cita demuestra que Carpentier no elude ningún clisé para denunciar a los surrealistas. A parte de la supuesta mancha de ser intelectuales empedernidos, dados a la discusión como a un vicio, rencorosos y de carácter voluble, pecan de otras sperversiones no menos sgraves Para fundar su negación global, el autor los pone en una fila con ninfómanas y lesbianas, trotskistas, anarquistas y adictos al psicoanálisis freudiano (๖[...] todas más o menos abonadas al mágico diván de Don Segismundo $)$ [...]. Cualquier lector que recuerde, por ejemplo, los tópicos del discurso franquista, aqui cree haberse equivocado de libro [...].« 
nifiestos de corrillo; allá, allá, disparaban los máusers sobre quienes firmaban manifiestos, dejando cadáveres en las escalinatas universitarias [...]. Y eran numerosísimos los estudiantes cubanos que ahora se encontraban en París, por huir de la represión machadista, o tratando de proseguir sus estudios interrumpidos, allá, por los atropellos de la dictadura. (CP, 78)

Paris, die kalte und, im Gegensatz zu La Habana, menschenfeindliche und die Kommunikation unterbindende Hauptstadt aller künstlerischen Avantgardebewegungen, sei der Schauplatz eines Kulturbetriebs, der sich den Luxus einer neuen Romantik mit allen nur denkbaren Absurditäten leiste. Den europäischen Künstlern und Intellektuellen wird ein unverantwortlich oberflächliches und subjektivistisches Verhalten vorgehalten. Europa habe seine ganze Tradition und Bildung über Bord geworfen und verliere sich in Banalitäten. Und der Anführer und das Sprachrohr dieser Bande von Nichtsnutzen heißt André Breton. Ihm wird mit Vicente Huidobro (und Alfonso Reyes) der universal gebildete und ernsthafte lateinamerikanische Künstler und Intellektuelle gegenübergestellt. So wandere das, was an Wertvollem an der europäischen Kultur fortzudauern verdient in einer Art neuer translatio studii nach Amerika aus, wo ihr in Kuba neue Entfaltungsmöglichkeiten geboten würden. Unter allen Romanen Carpentiers ist $L a$ Consagración de la Primavera der am meisten ideologisch geprägte und eine geradezu realitätsblinde Panegyrik und Apologie des kubanischen Regimes. Was Carpentier an Breton kritisiert, kennzeichnet viel eher ihn selbst: das halsstarrige Verharren auf nicht in Frage zu stellenden Überzeugungen, die fehlende Bereitschaft, sich auf das Andere, das Fremde sine ira et studio einzulassen und eine erstaunliche Oberflächlichkeit, die der ostentative bildungsbürgerliche Enzyklopädismus, den er ausbreitet, nicht zu kaschieren vermag.

\section{IV.}

Mit Los Pasos perdidos (1953) setzt Carpentier sein Projekt fort, die Chronik der wunderbaren Wirklichkeit Lateinamerikas zu schreiben. ${ }^{6}$ Konzentrierte er sich in El Reino de este Mundo auf die Geschichte Lateinamerikas aus der Sicht eines versklavten und unterdrückten Afroamerikaners und auf die Kraft, die Stärke, die Vitalität der in Lateinamerika weiterlebenden afri-

6 Siehe hierzu besonders Claudius Armbruster (1982). 
kanischen Kultur, so konzipiert er, ähnlich wie fast gleichzeitig Pablo Neruda seinen Canto General, Los Pasos perdidos als umfassendes Epos über Lateinamerika. Beide, Neruda und Carpentier, kannten ja kaum etwas von Lateinamerika, als sie in den zwanziger Jahren ihre Heimatländer verließen und fast ohne Unterbrechung bis zum Ausbruch des Zweiten Weltkriegs fern des Subkontinents lebten. Und beide beginnen sie nach der Rückkehr ihre Entdeckungsreisen, realiter und in den Bibliotheken. Für beide ist schließlich das Schreiben in ihrer persönlichen Erfahrung verwurzelt. Dies gilt für die Entstehung von El Reino de este Mundo - Carpentier reist 1943 nach Haiti - ebenso wie die von Los Pasos perdidos - er unternimmt 1947/1948 eine Expedition ins Orinokogebiet.

Die schönsten und überzeugendsten Abschnitte dieses programmatisch, thematisch und intertextuell völlig überfrachteten `totalen Romans über das real maravilloso Lateinamerikas, in Raum und Zeit wie in Geschichte und Gegenwart, schildern die grandiose und ursprüngliche Natur des tropischen Urwalds. Diese »selva virgen« verkörpert in Los Pasos perdidos vor allen Dingen die Größe und Vitalität der Neuen Welt. Auch hier trifft sich Carpentier mit Neruda; er unterscheidet sich von ihm allerdings in der Wahrnehmungsweise des Protagonisten, dem sich eine solche Fülle von Assoziationen aus der europäischen literarischen und künstlerischen Tradition des Okzidents aufdrängt, dass die Unmittelbarkeit der Erfahrung des Anderen und des Fremden sich nur mit Mühe und nach geraumer Zeit einstellt.

Auch auf dieser Reise zurück zu den Ursprüngen, zur Erschaffung der Welt ebenso wie zur künstlerischen Schöpfung, begegnen wir der Dichotomie von Europa und Lateinamerika. ${ }^{7}$ Jetzt, nach dem Zweiten Weltkrieg und nach der ungeheuerlichen Barbarei des Nationalsozialismus ist Europa, ist das christliche Abendland, so eine der zentralen Aussagen des Romans, als Leitbild und als moralische Instanz endgültig untergegangen. Diese suggestive Gleichsetzung Europas mit der rassistischen Ideologie und mit dem Völkermord des Nationalsozialismus treibt die Europakritik auf die Spitze. Ein ähnlich mörderischer und unmenschlicher Rassismus hatte doch auch die europäischen Eroberer - die Spanier und Portugiesen, die Franzosen, die Deutschen, die Engländer und die Holländer - in Amerika ihre Untaten

7 Unter der Fülle der Arbeiten über Los Pasos perdidos hebe ich den konzentrierten Artikel von Friedrich Wolfzettel (1992) hervor. 
ohne Skrupel begehen lassen. Was zählt dagegen schon >das bisschen Barbarei in Lateinamerika, womit - im Roman - die Reisenden, kaum in Südamerika angekommen, sofort in Gestalt eines eben ausbrechenden Militärputsches konfrontiert werden? Dieser Golpe vollzieht sich so klischeehaft wie auf der Bühne eines Operettentheaters.

Die unübersehbare Schwäche von Los Pasos perdidos besteht darin, dass der Roman Carpentiers Ideen über Lateinamerika illustrieren soll. Diese literarische Konzeption übernimmt er von den französischen Existenzialisten. Gegen die in Paris und in Europa grassierende, in seinen Augen für die intellektuelle und künstlerische Dekadenz Europas charakteristische snihilistische< Mode des Existenzialismus polemisiert er in Los Pasos perdidos ganz besonders vehement; denn er gibt Mouche, der Geliebten des Erzählers, die diesen auf seiner Reise zu seinen amerikanischen Wurzeln anfangs begleitet, die Züge einer arroganten, oberflächlichen, selbstgefälligen und der kraftvollen Natur Amerikas nicht gewachsenen existenzialistischen Intellektuellen, die sich im »großen Trödelladen des Surrealismus gebildet« habe: $»[\ldots]$ se había formado intelectualmente en el gran baratillo surrealista (PP, 146). Die Existenzialistin Mouche repräsentiert Europa in exakt derselben negativen Weise wie Breton und Descartes. Kein Wunder, dass sie drei jungen, ausschließlich auf Europa und auf die europäische Kunst fixierten lateinamerikanischen Künstlern begeistert applaudiert, während der Erzähler die echte Kunst Lateinamerikas in einer Kneipe antrifft ${ }^{8}$, wo ein anonymer, aus einem entlegenen Dorf stammender Harfenspieler aus der Tiefe der volkstümlichen Traditionen zu musikalischen Ergebnissen kommt, die den Versuchen der Avantgardekünstler der Gegenwart in nichts nachstehen - »alcanzándose, por los caminos de un primitivismo verdadero, las búsquedas más válidas de ciertos compositores de la época presente« (PP, 198).

Wie Mouche ist auch ihre Gegenspielerin Rosario die Verkörperung einer Denkfigur oder einer Idee: In ihrem Namen, in ihrer Erscheinung und

8 Diese strukturelle Opposition von Künstlerstudio (hier das Atelier einer Malerin) und dem lebendigen Musikgenuss in einer Kneipe taucht in La Consagración de la Primavera wieder auf: Im Keller des Hauses, in dem sich Bretons Wohnung befindet - »el Gran Laboratorio Central del Surrealismo « (CP, 85), hat sich die Cabaña Cubana eingenistet, wo der Erzähler so richtig versinken kann in den Rhythmen der kubanischen, der karibischen, der afroamerikanischen Musik. An diesem Beispiel zeigt sich besonders anschaulich, wie strukturell und topologisch Carpentier denkt und schreibt. Es handelt sich um ein fest gegründetes Gedankengebäude, das er allen seinen Romanen, Essays, Vorträgen und Interviews zu Grunde legt und über die Jahre hinweg fast unverändert beibehält. 
in ihrem Verhalten manifestiert sich der ethnische und kulturelle mestizaje Lateinamerikas. ${ }^{9}$ Als sich der Erzähler in einem »abrazo rápido y brutal « (PP, 278-280) mit Rosario vereinigt, beginnt das eigentliche Eindringen in die andere und fremde Welt Lateinamerikas und zugleich der ebenso brutale Bruch mit dem Okzident, mit Mouche. Diese sanimalische< Liebesszene zeigt, dass jene Stelle aus dem Vorwort zu El Reino de este mundo, in der die Vergewaltigung lebendiger statt toter Frauen empfohlen wird, Carpentier nicht versehentlich unterlaufen ist, sondern einem Frauenbild entspricht, in dem sich die erotische Attraktivität der amazonenhaften Wildheit der ursprünglichen und naturverbundenen, tellurischen >Eingeborenen< mit der von der Natur festgelegten Funktion der Frau als der Gebärerin und Mutter, die den Samen des Mannes aufnimmt, verbindet. ${ }^{10}$ Hier offenbart Carpentier einen sexistischen Exotismus, der zum festen Inventar des kolonialistischen Diskurses gehört.

Abschließend sei darauf hingewiesen, dass Carpentier bereits mit der Wahl des Titels Los Pasos perdidos einen intertextuellen Hinweis darauf gibt, wie grundsätzlich er sich auch mit diesem Roman von Breton und dem Surrealismus distanziert. Breton veröffentlicht 1924, unmittelbar vor der Publikation des Manifeste du Surréalisme eine Sammlung von 24 literatur- und kunstkritischen Essays, die, von einer Ausnahme abgesehen, zwischen 1918 und 1923 entstehen und in verschiedenen Zeitschriften erscheinen, unter dem Titel Les Pas perdus. Er erzählt dort von seiner Begegnung mit Jacques Vaché, der ihn außerordentlich beeindruckt habe mit seiner radikalen Art, Kunst zu leben, er spricht über Apollinaire, Jarry, Lautréamont und Aloysius Bertrand, über Max Ernst, Giorgio De Chirico, Marcel Duchamp und Francis Picabia und fasst so die Entwicklung seines Weges vom Dadaismus zum Surrealismus zusammen. Gemeinsam ist allen Arbeiten die Frage nach dem Sinn der Kunst, vor allem der Dichtung, und nach dem Verhältnis von Kunst und Leben. ${ }^{11}$ Eine unvoreingenommene vergleichende Lektüre von Les Pas perdus und dem Vorwort zu El Reino de este Mundo geht, wie mir scheint, eindeutig zu Gunsten von Breton aus,

9 Siehe hierzu und zum Folgenden Karl Hölz (1998: 189-195).

10 Die Liebesszene endet mit folgendem Satz: "Me he sembrado bajo el vellón que acaricio con mano de amo, y mi gesto cierra una gozosa confluencia de sangres que se encontraron« (PP, 280).

11 Cf. André Breton (1988: 1219). 
dessen subtile und keineswegs >blutlose $<$ Argumentation Carpentiers polemisch vorgetragenes ästhetisches Programm ins Leere laufen lässt. ${ }^{12}$

$$
\text { V. }
$$

Carpentier malt in seinen Romanen ein durch und durch negatives Bild von Europa. Sein Europa-Topos wird gebildet aus Eurozentrismus, Kolonialismus, Imperialismus, Inauthentizität, aus organisierter Barbarei und aus moralischer, intellektueller sowie künstlerischer Dekadenz. Nur dann, wenn Europa an seine barocke Tradition anknüpfe und wenn es die kulturellen Einflüsse der >Dritten Welt $<(\mathrm{CP}, 86)$ zustimmend aufgreife, könne es zu neuer Lebendigkeit zurückfinden (wie etwa in Concierto barroco veranschaulicht). Er als Lateinamerikaner und als poeta doctus setze die kulturelle Mission Europas fort; denn deren zentrale Begriffe - Bildung, Aufklärung, Moral, Vitalität, politisches Bewusstsein - hätten sich nach Lateinamerika verlagert (translatio studii).

Dieser topischen Europa-Kritik entspricht die systematische Hypostasierung Lateinamerikas: Seine Natur, seine Menschen, seine Kultur und Kunst seien unerhört vital und durch den mestizaje überreich. Hier stehe der Künstler vor gewaltigen Aufgaben, hier habe das künstlerische Schaffen einen wirklich kreativen Sinn, da der Künstler ein neuer Adam sei, der die chaotische Welt benennen und ordnen müsse.

Dieses voluntaristische, ideologische und exotistische LateinamerikaBild entbehrt heute jeder Grundlage. Gewiss ist es kein Zufall, dass Los Pasos perdidos sich auch erst nach der begeisterten Rezeption in Frankreich in Lateinamerika durchsetzte. ${ }^{13}$ Die europäischen Leser fanden und finden sich offensichtlich in ihren Erwartungen, Vorurteilen oder exotistischen Projektionen aufs Schönste bestätigt.

Es soll nun aber zum Schluss dieser Kritik zweierlei angemerkt werden: Erstens hat sich Carpentier in den etwa viertausend Artikeln, die er zwischen 1951 und 1959 in Caracas in der Zeitung El Nacional unter dem Ko-

12 Wenigstens en passant soll darauf hingewiesen werden, dass ein anderer berühmter Schriftsteller aus der Karibik, der zur gleichen Zeit wie Carpentier in Paris mit dem Surrealismus in Berührung kommt und ihm zunächst ebenfalls skeptisch begegnet, später ein fruchtbares Verhältnis zu Breton entwickeln wird: Ich meine Aimé Césaire, dessen Cahier d'un Retour au Pays natal immer wieder in die Nähe zum Surrealismus gerückt wird und dessen Zusammentreffen mit Breton 1941 auf Martinique überaus positiv verläuft.

13 Cf. Friedrich Wolfzettel (1992: 244). 
lumnen-Titel »Letra y Solfa« über Literatur und Musik publizierte, sehr differenziert und kenntnisreich über die literarischen, musikalischen, ästhetischen und kulturellen Entwicklungen Europas geäußert. Hier bemüht er sich um eine ernsthafte und fruchtbare Vermittlung zwischen den Kontinenten. Und zweitens verdanken wir ihm eine ganze Reihe von Werken, die weiter Bestand haben werden. Dazu rechne ich vor allem seine frühen Erzählungen, den großen Roman über die Dialektik der Aufklärung und ihre Auswirkungen auf die Karibischen Inseln - El Siglo de las Luces - und die witzige und ironische Europareise in Concierto barroco.

\section{Bibliographie}

\section{Literarische Werke und andere Quellen}

Breton, André (1988): Les Pas perdus (1924), in: id.: Euvres complètes, hrsg. v. Marguerite Bonnet, Paris: Gallimard, Bd. 1, pp. 191-308, 1216-1332.

Carpentier, Alejo (1983): Obras completas, Mexiko: Siglo XXI, Bd. II: El Reino de este Mundo und Los Pasos perdidos.

Carpentier, Alejo (1984): Obras completas, Mexiko: Siglo XXI, Bd. VI: El Recurso del Método.

Carpentier, Alejo (1978): Obras completas, Mexiko: Siglo XXI, Bd. VII: La Consagración de la Primavera.

Roa Bastos, Augusto (1974): Yo el Supremo, Buenos Aires: Siglo XXI.

Vargas Llosa, Mario (2000): La Fiesta del Chivo, Madrid: Alfaguara.

Forschungsliteratur

Armbruster, Claudius (1982): Das Werk Alejo Carpentiers. Chronik der »Wunderbaren Wirklichkeit«, Frankfurt am Main: Vervuert.

Casanova, Pascale (1999): La République mondiale des Lettres, Paris: Seuil.

Garscha, Karsten (1991): »Alejo Carpentiers Verhältnis zur europäischen, besonders zur französischen Avantgarde«, in: Wentzlaff-Eggebert, Harald (Hrsg.): Europäische Avantgarde im lateinamerikanischen Kontext. Akten des internationalen Kolloquiums 1989, Frankfurt am Main: Vervuert, pp. 511-520.

Garscha, Karsten (1996): „Auf der Höhe der Zeit. Michael Rössner ediert seine Lateinamerikanische Literaturgeschichte«, Frankfurter Rundschau 82 (6.4.1996), ZB 4.

Geisler, Eberhard (1985): »La subjetividad sacrificada. Apuntes para leer La consagración de la primavera de Alejo Carpentier«, in: Bremer, Thomas / Losada, Alejandro (Hrsg.): Aclas de la Asociación de Estudios de Literaturas y Sociedades de América Latina 1983-1984. Hacia una historia social de la literatura latinoamericana, Gießen: AELSAL, pp. 129-139.

Hölz, Karl (1998): Das Fremde, das Eigene, das Andere. Die Inszenierung kultureller und geschlechtlicher Identität in Lateinamerika, Berlin: Schmidt. 


\section{Karsten Garscha}

Janik, Dieter (1976): Magische Wirklichkeitsauffassung im hispanoamerikanischen Roman des 20. Jahrhunderts, Tübingen: Niemeyer.

Janik, Dieter (1992): Stationen der spanischsprachigen Literatur- und Kulturgeschichte: der Blick der anderen - der Weg zu sich selbst, Frankfurt am Main: Vervuert.

Wolfzettel, Friedrich (1992): »Alejo Carpentier: Los Pasos perdidos«, in: Roloff, Volker / Wentzlaff-Eggebert, Harald (Hrsg.): Der hispanoamerikanische Roman, Darmstadt: Wissenschaftliche Buchgesellschaft, Bd. 1, pp. 244-253, 362-363. 